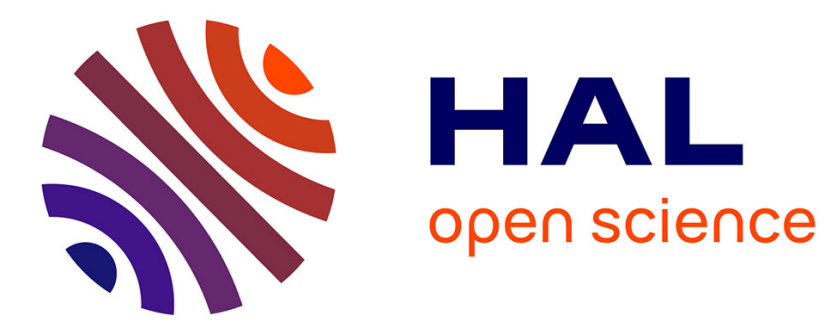

\title{
Photothérapies du psoriasis
}

\author{
J.-C. Béani, M. Jeanmougin, J.-L. Schmutz
}

\section{To cite this version:}

J.-C. Béani, M. Jeanmougin, J.-L. Schmutz. Photothérapies du psoriasis. Annales de Dermatologie et de Vénéréologie, 2019, 146, pp.450 - 453. 10.1016/j.annder.2019.04.007 . hal-03487901

\section{HAL Id: hal-03487901 \\ https://hal.science/hal-03487901}

Submitted on 20 Dec 2021

HAL is a multi-disciplinary open access archive for the deposit and dissemination of scientific research documents, whether they are published or not. The documents may come from teaching and research institutions in France or abroad, or from public or private research centers.
L'archive ouverte pluridisciplinaire HAL, est destinée au dépôt et à la diffusion de documents scientifiques de niveau recherche, publiés ou non, émanant des établissements d'enseignement et de recherche français ou étrangers, des laboratoires publics ou privés.

\section{다)(1) $(5$}

Distributed under a Creative Commons Attribution - NonCommercial| 4.0 International 


\section{PHOTOTHERAPIES DU PSORIASIS \\ PHOTOTHERAPY FOR PSORIASIS}

\section{J.-C. Béani ${ }^{1}$, M. Jeanmougin ${ }^{2}$, J.-L. Schmutz ${ }^{3 *}$ \\ et Groupe de recherche sur le Psoriasis de la Société Française de Dermatologie}

1 : Professeur honoraire - 163, chemin Sainte Claire, 38340 Voreppe, France

2: Service de Dermatologie, Hôpital Saint Louis, 1 Avenue Claude Vellefaux, 75010 Paris, France

3 : Service de Dermatologie, Hôpitaux de Brabois, CHRU de Nancy, 54500 Vandœuvre-lèsNancy, France

* Auteur correspondant.

Adresse e-mail : jl.schmutz@chru-nancy.fr (J.-L. Schmutz)

Conflits d'intérêts : Les auteurs déclarent n'avoir aucun lien d'intérêt. 
Deux photothérapies, PUVA (Psoralène et UltraViolet A) et UVB à spectre étroit, sont principalement utilisées dans la prise en charge du psoriasis dans les formes modérées à sévères en plaques ou en gouttes.

\section{- Mode d'action}

L'exposition de la peau aux UV, utilisés soit seuls soit en association à un photosensibilisant (psoralène), entraîne, via des réactions photochimiques directes ou photosensibilisées, des effets biologiques variés, bénéfiques pour une peau pathologique. Pour le psoriasis, sont essentiellement concernés l'effet anti-prolifératif et l'effet immunomodulateur.

\section{- Indication}

L'indication est retenue en fonction de la sévérité et de l'étendue de la poussée du psoriasis, des conséquences psychologiques de la dermatose, du rapport bénéfice/risque par rapport aux autres possibilités de traitement, de la disponibilité du patient (facteur essentiel de l'observance thérapeutique), du nombre de séances cumulées lors des cures précédentes et de l'absence de contre-indications absolues et relatives.

\section{- Contre-indications}

Absolues: syndrome des naevus dysplasiques héréditaires; antécédent personnel de mélanome; lupus érythémateux systémique; maladies avec troubles de la réparation de l'ADN.

Relatives majeures : antécédents de carcinome cutané ; exposition antérieure aux radiations ionisantes ou à l'arsenic ; présence de kératoses actiniques ; traitement immunosuppresseur concomitant; porphyries.

Contre-indications relative mineures : âge inférieur à 8 ans; pemphigoïde; pemphigus ; traitement antérieur par le méthotrexate ou la ciclosporine ; photosensibilité cutanée, liée ou non à des médicaments photosensibilisants (le plus souvent aux UVA); sujet présentant de nombreux naevi ( $>50$ ); sujet présentant plus de 5 naevus atypiques

Contre-indications du 8 métoxypsoralène (Méladinine ${ }^{\circledR)}$ : enfant ; grossesse ; allaitement ; hypertension artérielle ; insuffisance cardiaque ; insuffisance hépatique ou rénale ; cataracte.

\section{- Posologie et modalités pratiques de prescription}

Le principe de la PUVA nécessite préalablement à l'exposition aux UVA l'administration d'un psoralène (Méladinine®), le plus habituellement faite par voie orale $(0,6 \mathrm{mg} / \mathrm{kg}$ à prendre 2 heures avant l'exposition aux UVA) ou locale (à appliquer 20-30 minutes sur les 
lésions à traiter avant l'exposition aux UVA), mais pouvant aussi se faire par l'immersion d'un segment de membre ou du corps entier dans une solution aqueuse de Méladinine ${ }^{\circledR}$ (balnéoPUVA) (1). Une protection oculaire et des organes génitaux externes est recommandée. La photothérapie UVB ne nécessite pas de prise préalable de psoralène.

- L'exposition corporelle aux $U V$ est réalisée grâce à des cabines d'irradiation équipées soit de tubes Philips UVB TL01, soit de tubes UVA (Philips TL09 ou Sylvania dite PUVA), soit des deux (21 tubes UVB et 21 tubes UVA), homologuées (marquage CE, certification ISO-DIN) et munies de systèmes de dosimétrie performants. Des modules adaptés permettent aussi une exposition localisée des mains, des pieds ou du cuir chevelu .

- Le rythme des séances est habituellement de 3 par semaine, espacées de $48 \mathrm{~h}$ pour la PUVA ; les séances UVB peuvent être réalisées tous les jours de façon consécutive; plusieurs protocoles sont validés et utilisables pour l'augmentation des doses UV au cours des séances successives. Une fois le résultat acquis, l'intérêt d'un traitement d'entretien n'est pas confirmé.

A l'attention des patients sont disponibles des fiches d'information et des fiches de consignes pour la PUVAthérapie orale et la photothérapie UVB. Il est possible d'accéder à ces fiches sur le site du Groupe de Recherche sur le Psoriasis de la Société Française de Dermatologie (www.grpso.org).

La prise en charge par la sécurité sociale du traitement suppose une entente préalable (Cerfa 60-3584). La cotation CCAM ne fait pas de différence entre PUVA orale et UVB TL01 mais cote différemment selon la surface traitée, corporelle totale (QZRP003) ou localisée (QZRP002), et en cas de balnéoPUVA, localisée (QZRP005) ou générale (QZRP004).

\section{- Bilan initial $(2,3)$}

Un examen clinique complet recherchera des contre-indications ou des éléments imposant des explorations complémentaires avant la décision finale de traiter. Il comportera un interrogatoire pour exclure, notamment, l'usage de médicaments photosensibilisants. Le consentement éclairé au traitement du patient doit être obtenu et une fiche d'information validée doit lui être remise (4).

\section{- Efficacité attendue}

L'amélioration est nette habituellement, entre la 10ème et la 20ème séance. 
La PUVA orale reste la référence pour une poussé étendue avec un blanchiment complet, ou quasi complet (PASI 90) dans 80 à $90 \%$ des cas après 15 à 25 séances; la balnéoPUVAthérapie paraît particulièrement intéressante chez les sujets à phototype foncé. Elle est préférable en première intention dans les psoriasis étendus sévères en grandes plaques épaisses (niveau de preuve A), chez les adultes de phototype IV à VI (niveau de preuve B) ; elle sera aussi être envisagée pour les psoriasis résistants aux UVB-TL01 (niveau de preuve B).

La photothérapie UVB à spectre étroit est également très efficace chez 60 à $90 \%$ des patients en 20 à 40 séances (niveau de preuve A); cependant les résultats sont nettement meilleurs dans les psoriasis en gouttes ou nummulaires que dans les psoriasis à grandes plaques. Elle représente une bonne alternative à la PUVAthérapie par l'absence de prise de psoralène, par un faible taux d'effets secondaires immédiats et une réduction du risque de photosensibilisation médicamenteuse intercurrente, par l'absence de nécessité d'une photoprotection cutanée et oculaire après la séance $(5,6)$.

L'efficacité des photothérapies localisées pour les psoriasis des mains et des pieds est moins importante (50\% des patients très améliorés en 30 à 40 séances) surtout dans les formes très kératosiques.

\section{- Effets indésirables}

On distingue les effets indésirables précoces, essentiellement l'érythème phototoxique lié à une erreur de dosage UV ou à la prise concomitante de produits photosensibilisants (médicaments, aliments, phytothérapie, etc...).

Seuls les effets indésirables tardifs restent un problème (7).

- Le risque potentiel de favoriser une cataracte impose une protection oculaire pendant les séances et pendant les 12 heures suivant la prise de psoralène pour la PUVA. En l'absence de symptomatologie ou d'affection oculaire connue, il n'est pas recommandé d'effectuer un contrôle ophtalmologique avant de débuter une photothérapie.

- Le risque cancérigène de la photothérapie est dépendant de la dose d'UV reçue. Pour la PUVA, ce risque est établi et quantifié grâce à des études de cohorte fiables; un seuil maximal de 200 séances a été proposé. Pour les UVB à spectre étroit, ce risque n'est actuellement pas quantifié de manière précise. Il parait raisonnable de considérer qu'au-delà de 250 séances de photothérapie (PUVA et/ou UVB spectre étroit), il existe un risque accru de cancers cutanés chez certaines personnes. Le risque cancérigène de la photothérapie est majoré très significativement chez les patients ayant été aussi traités par ciclosporine. Il 
n'existe aucune donnée actuelle sur le risque carcinogène chez les patients sous biothérapies et ayant été aussi traités par photothérapies. Il apparait souhaitable de faire une surveillance dermatologique attentive chez les patients ayant reçu plus de 150 séances de photothérapie et traités par biothérapie.

\section{- Surveillance sous traitement}

Une surveillance dermatologique avant chaque séance est recommandée pour éviter le risque de surdosage et adapter la dose d'UV prescrite. Une surveillance dermatologique annuelle est recommandée en raison du risque carcinogène $(2,8)$.

\section{- Circonstances particulières}

La PUVA thérapie est contre-indiquée chez les enfants et pendant la grossesse et l'allaitement. La photothérapie UVB est recommandée comme photothérapie de première intention (niveau de preuve A), chez l'enfant et l'adolescent, et chez l'adulte dans les psoriasis étendus modérés en petites plaques superficielles. Elle est également utilisable, contrairement à la PUVA, chez la femme enceinte ou allaitante et en cas d'insuffisance rénale ou d'insuffisance hépatique. Elle aura aussi la préférence chez le sujet VIH positif (niveau de preuve C).

\section{- Traitements associés à la photothérapie}

Leur but est double, réduire les effets secondaires (en réduisant notamment le nombre de séances) et augmenter l'efficacité des photothérapies. Le décapage des lésions doit être obtenu avant le début de la photothérapie, notamment en utilisant de la vaseline. Un effet synergique a été démontré avec les dermocorticoïdes et le tazarotène, moins nettement avec les dérivés topiques de la vitamine D3. Quand il n'existe pas de contre-indication à sa prescription, l'acitrétine a démontré un bénéfice synergique en association avec la PUVA et avec la photothérapie UVB TL01. 


\section{Références}

1. Schmutz J.L, Jeanmougin M, Martin S, Amblard P, Thomas P. Recommandations de la Société française de photodermatologie pour la PUVAthérapie systémique dans le psoriasis vulgaire. Ann Dermatol Venereol $2000 ; 127$ : 753 - 9.

2. Amatore F, Villani AP, Tauber M, Viguier M, Guillot B;Psoriasis Research Group of the French Society of Dermatology (Groupe deRecherche sur le Psoriasis de la Société Française de Dermatologie).French guidelines on the use of systemic treatments for moderate-to-severe psoriasis in adults. J Eur Acad Dermatol Venereol. 2019 Feb 22. doi: $10.1111 /$ jdv.15340.

3. Richard MA. Psoriasis : évaluation initiale et bilan thérapeutique pratique. Ann Dermatol Venereol 2019. 10.1016/j.annder.2019.03.006

4. Menter A, Korman NJ, Elmets CA, Feldman SR, Gelfand JM, Gordon KB, et al. Guidelines of care for the management of psoriasis and psoriatic arthritis: Section 5. Guidelines of care for the treatment of psoriasis with phototherapy and photochemotherapy. J Am Acad Dermatol 2010;62:114-35.

5. Mehta D, Lim HW. Ultraviolet B Phototherapy for Psoriasis: Review of Practical Guidelines. Am J Clin Dermatol 2016;17:125-33.

6. Beani JC, Jeanmougin M. La photothérapie UVB spectre étroit dans le psoriasis vulgaire : utilisation pratique et préconisations de la société française de photodermatologie. Ann Dermatol Venereol. 2010;137:21-31.

7. Beani JC. Photothérapies et photochimiothérapies par ultraviolets, EMC (Elsevier SAS, Paris) [98-930-A-10] - Doi : 10.2016/S0246-0319(16)69012-X.

8. Vassantachart JM, Soleymani T, Wu JJ. Comparison of phototherapy guidelines for psoriasis : a critical appraisal and comprehensive review. J Drugs Dermatol 2016;15:995-1000. 


\section{0 points pratiques avec la photothérapie au cours du psoriasis}

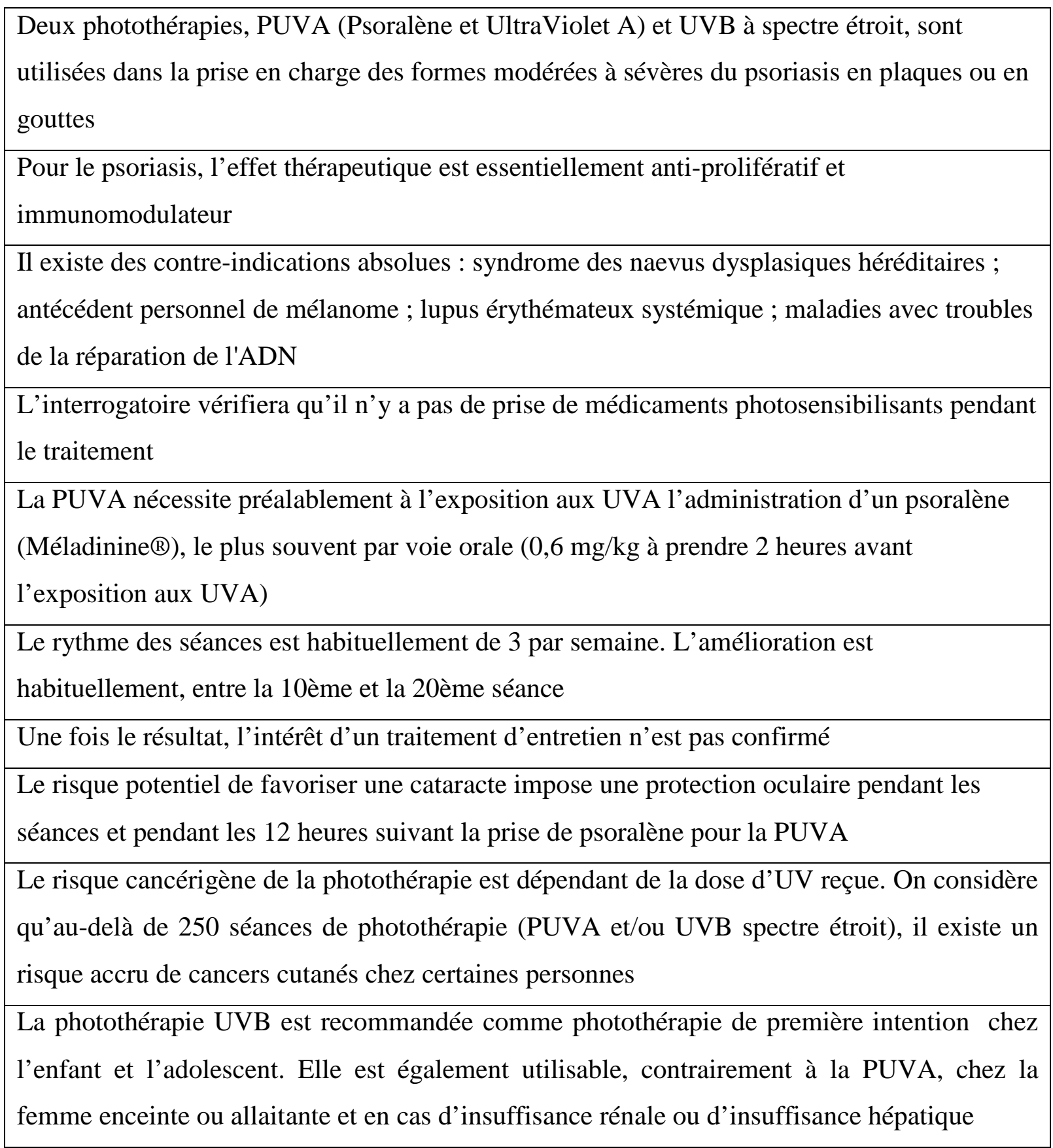

